\title{
The Skew Generalized Secant Hyperbolic Family
}

\author{
Matthias Fischer \\ Department of Statistics and Econometrics, Erlangen-Nürnberg
}

\begin{abstract}
We introduce a skewness parameter into Vaughan's (2002) generalized secant hyperbolic (GSH) distribution by means of exponential tilting and develop some properties of the new distribution family. In particular, the moment-generating function is derived which ensures the existence of all moments. Finally, the flexibility of our distribution is compared to similar parametric models by means of moment-ratio plots and application to foreign exchange rate data.
\end{abstract}

Keywords: Hyperbolic Secant Distribution, Skewness, Esscher Transformation.

\section{Introduction}

Recently, Vaughan (2002) suggested a family of symmetric distributions-the so-called generalized secant hyperbolic (GSH) distribution-with kurtosis ranging from 1.8 to infinity. This family includes both hyperbolic secant and logistic distribution, and closely approximates the Student $t$-distribution with corresponding kurtosis. In addition, the moment-generating function and all moments exist, and the cumulative distribution is given in closed form. Unfortunately, this family does not allow for skewness. For this purpose, a skew version is developed in section 2 which maintains many properties of the GSH family derived in section 3. To demonstrate its flexibility, both moment-ratio plot and goodness-of-fit are compared to those of similar successful parametric models, like the EGB2 distribution (see, e.g., McDonald, 1984) or the skew generalized error (SGED) distribution (see, e.g. Theodossiou, 1998) in Section 4.

\section{Definition}

Vaughan (2002, p. 221) introduces the (symmetric) generalized secant hyperbolic (GSH) distribution with probability density function

$$
f(x ; t) \equiv\left\{\begin{array}{lr}
\frac{\sin (t)}{t} \exp (x)[\exp (2 x)+2 \cos (t) \exp (x)+1]^{-1}, & -\pi<t<0, \\
\exp (x)[\exp (2 x)+2 \exp (x)+1]^{-1}, & t=0 \\
\frac{\sinh (t)}{t} \exp (x)[\exp (2 x)+2 \cosh (t) \exp (x)+1]^{-1}, & t>0,
\end{array}\right.
$$

where the parameter $t>-\pi$ governs the amount of kurtosis. In particular, the usual coefficient of kurtosis $\mathbb{K}(t)$-i.e. the fourth standardized moment of $X$-comprises the interval $] 1.8, \infty[$ with $\mathbb{K}(0)=4.2$ which corresponds to the logistic distribution. Moreover, setting $t \equiv-\pi / 2$, the hyperbolic secant distribution is obtained. Vaughan (2002) also notes that as $t \rightarrow \infty$, the density tends to that of the standard uniform distribution 
on $(-\sqrt{3}, \sqrt{3})$. Despite its flexibility, the GSH distribution is not able to account for skewness.

To remove this shortcoming though pertaining many of the attractive properties, we apply the Esscher transformation to $X$. Originally, the concept of the Esscher transformation was a time-honored tool in actuarial science suggested by Esscher (1932) which became popular by Gerber and Shiu (1995) who apply this concept to value derivative securities. Assuming that the moment-generating function $\mathcal{M}(u)=\mathrm{E}(\exp (u X))$ of a variable $X$ with density $f(x)$ exists, the Esscher-transformed density is defined by

$$
f(x ; h) \equiv \exp (h x) f(x) / \mathcal{M}(h) .
$$

Note that if the Esscher transformation is applied to is a Gaussian variable the resulting variable is again Gaussian (and thus symmetric) but with different parameters. In contrast, Esscher-transformations of symmetric non-Gaussian densities result in asymmetric distributions, where $h$ governs the amount of skewness and symmetry is obtained for $h=0$. Combining (1) and (2), and noting that the moment-generating function of a GSH variable (see Vaughan, 2002, p. 221) is given by

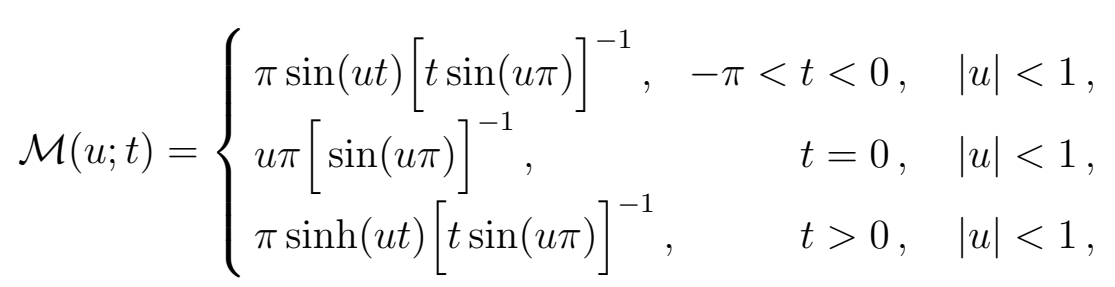

the Esscher-transformed GSH density for $-\pi<t<0$ and $h \neq 0$ derives as

$$
f(x ; t, h) \equiv \frac{\sin (h \pi) \sin (t)}{\pi \sin (h t)} \frac{\exp ((h+1) x)}{\exp (2 x)+2 \cos (t) \exp (x)+1} .
$$

Similarly, for $t>0$ and $h \neq 0$,

$$
f(x ; t, h)=\frac{\sin (h \pi) \sinh (t)}{\pi \sinh (h t)} \frac{\exp ((h+1) x)}{\exp (2 x)+2 \cosh (t) \exp (x)+1},
$$

and for $t=0$ and $h \neq 0$,

$$
f(x ; 0, h)=\frac{\sin (h \pi)}{h \pi} \frac{\exp ((h+1) x)}{\exp (2 x)+2 \exp (x)+1} .
$$

Densities of the form (3), (4) or (5) will be termed as skew generalized secant hyperbolic densities, or briefly SGSH densities, in the sequel. Exemplarily, SGSH densities are plotted in Figure 1, below.

\section{Moment-Generating Function and Moment Ratio Plot}

We next show that the moment-generating function of a SGSH variable exists. Consequently, all moments exit, too. 

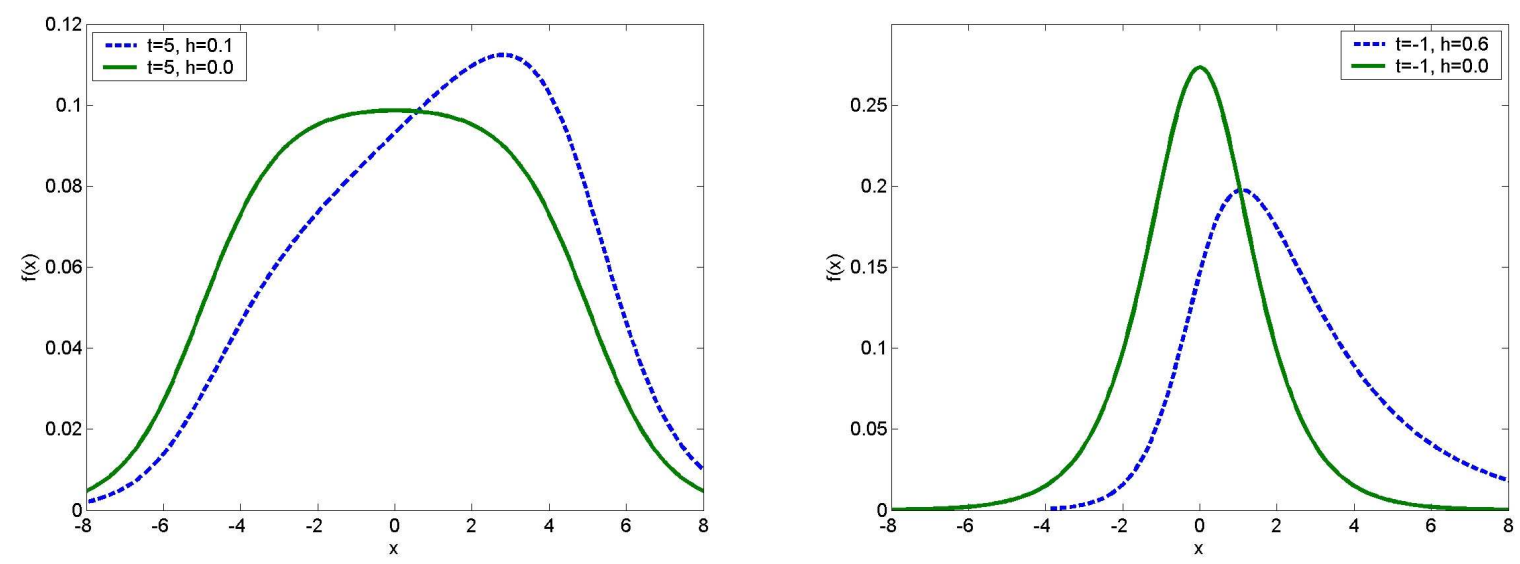

Figure 1: Skew GSH densities of type (a) Platykurtic (left), and (b) Leptokurtic (right).

Lemma 1 (Moment-generating function) The moment-generating function of a SGSH variable with $h \neq 0$ and $|u+t|<1$ is given by

$$
\mathcal{M}(u)=\left\{\begin{array}{cc}
\sin ((h+u) t) \sin (h \pi)[\sin ((h+u) \pi) \sin (h t)]^{-1}-\pi<t<0 \\
(h+u) \sin (h \pi)[h \sin ((h+u) \pi)]^{-1} & t=0 \\
\sinh ((h+u) t) \sin (h \pi)[\sin ((h+u) \pi) \sinh (h t)]^{-1} & t>0 .
\end{array}\right.
$$

In particular, all moments of the SGSH distribution exist.

Proof: Note that for $-\pi<t<0$ and $h \neq 0$,

$$
\begin{aligned}
& \mathcal{M}(u ; t, h)=\int \frac{\sin (h \pi) \sin (t)}{\pi \sin (h t)} \frac{\exp ((h+u+1) x)}{\exp (2 x)+2 \cos (t) \exp (x)+1} d x \\
= & \frac{\sin ((h+u) t)}{\sin ((h+u) \pi)} \frac{\sin (h \pi)}{\sin (h t)} \int \frac{\sin ((h+u) \pi) \sin (t)}{\pi \sin ((h+u) t)} \frac{\exp (((h+u)+1) x)}{\exp (2 x)+2 \cos (t) \exp (x)+1} d x .
\end{aligned}
$$

Similar reformulations hold for $t \geq 0$.

Lemma 2 (Power moments) Assume that $h \neq 0$. The first four power moments of a SGSH variable are given by

$$
\begin{gathered}
E(X)=\left\{\begin{array}{rr}
t \cot (h t)-\pi \cot (h \pi) & -\pi<t<0, \\
(1-h \pi \cot (h \pi)) / h & t=0, \\
t \operatorname{coth}(h t)-\pi \cot (h \pi) & t>0,
\end{array}\right. \\
E\left(X^{2}\right)=\left\{\begin{array}{lr}
\pi^{2}-t^{2}-2 t \pi \cot (h t) \cot (h \pi)+2 \pi^{2} \cot ^{2}(h \pi) & -\pi<t<0, \\
\pi^{2}-2 \pi / h \cot (h \pi)+2 \pi^{2} \cot ^{2}(h \pi) & t=0, \\
t^{2}+\pi^{2}-2 t \pi \operatorname{coth}(h t) \cot (h \pi)+2 \pi^{2} \cot ^{2}(h \pi) & t>0,
\end{array}\right.
\end{gathered}
$$




$$
\begin{gathered}
E\left(X^{3}\right)=\left\{\begin{array}{c}
-t^{3} \cot (h t)+3 t^{2} \pi \cot (h \pi)+6 t \pi^{2} \cot (h t) \cot ^{2}(h \pi)+3 t \pi^{2} \cot (h t) \\
-6 \pi^{3} \cot ^{3}(h \pi)-5 \pi^{3} \cot (h \pi), \quad-\pi<t<0, \\
6 \pi^{2} / h \cot ^{2}(h \pi)+3 \pi^{2} / h-6 \pi^{3} \cot ^{3}(h \pi)-5 \pi^{3} \cot (h \pi), \quad t=0, \\
t^{3} \operatorname{coth}(h t)-3 t^{2} \pi \cot (h \pi)+6 t \pi^{2} \operatorname{coth}(h t) \cot ^{2}(h \pi)+3 t \pi^{2} \operatorname{coth}(h t) \\
-6 \pi^{3} \cot ^{3}(h \pi)-5 \pi^{3} \cot (h \pi), \quad t>0,
\end{array}\right. \\
E\left(X^{4}\right)=\left\{\begin{array}{c}
t^{4}+5 \pi^{4}+4 t^{3} \pi \cot (h t) \cot (h \pi)-12 t^{2} \pi^{2} \cot ^{2}(h \pi)-6 t^{2} \pi^{2} \\
-24 t \pi^{3} \cot (h t) \cot ^{3}(h \pi)-20 t \pi^{3} \cot (h t) \cot (h \pi)+24 \pi^{4} \cot ^{4}(h \pi) \\
+28 \pi^{4} \cot ^{2}(h \pi), \quad t>0, \\
5 \pi^{4}-24 \pi^{3} / h \cot ^{3}(h \pi)-20 \pi^{3} / h \cot (h \pi)+24 \pi^{4} \cot ^{4}(h \pi) \\
+28 \pi^{4} \cot ^{2}(h \pi), \quad t=0, \quad \\
t^{4}+5 \pi^{4}-4 t^{3} \pi \operatorname{coth}(h t) \cot (h \pi)+12 t^{2} \pi^{2} \cot ^{2}(h \pi)+6 t^{2} \pi^{2} \\
-24 t \pi^{3} \operatorname{coth}^{2}(h t) \cot { }^{3}(h \pi)-20 t \pi^{3} \operatorname{coth}(h t) \cot (h \pi) \\
+24 \pi^{4} \cot ^{4}(h \pi)+28 \pi^{4} \cot ^{2}(h \pi), \quad-\pi<t<0 .
\end{array}\right.
\end{gathered}
$$

Note that the formulas of Lemma 2 can easily be worked out with the help of Maple or Mathematica. Moreover, the variance of a SGSH variable is given by

$$
\operatorname{var}(X)=\left\{\begin{array}{lr}
\pi^{2}\left(1+\cot ^{2}(h \pi)\right)-t^{2}\left(1+\cot ^{2}(h t)\right), \quad-\pi<t<0, \\
\pi^{2}\left(1+\cot ^{2}(h \pi)\right)-1 / h^{2}, & t=0, \\
\pi^{2}\left(1+\cot ^{2}(h \pi)\right)+t^{2}\left(1-\operatorname{coth}^{2}(h t)\right), & t>0 .
\end{array}\right.
$$

Similarly, Lemma 2 allows (tedious but straightforward) derivation of both skewness and kurtosis coefficient $\mathcal{S}(X)$ and $\mathcal{K}(X)$-i.e. third and fourth standardized moment - of a SGSH variable. In order to provide a visual assessment of skewness and kurtosis associated with a SGSH variable, moment ratio plots are a useful tool. These diagrams were introduced for Pearson-type distributions by Elderton and Johnson (1969). The classical moment ratio plot consists of all possible pairs $(\mathcal{S}(X), \mathcal{K}(X))$ that can be obtained through different combinations of the shape parameters of the underlying distribution (i.e. $t$ and $h$ for the SGSH distribution). In general, the relation $\mathcal{S}(X)^{2}<\mathcal{K}(X)+1$ for $\mathcal{K}(X)>0$ holds, i.e. for a given level of kurtosis only a finite range of skewness may be spanned. The moment ratio diagram of a SGSH variable was generated using random numbers from the domain of the shape parameters and is plotted in figure 2, below. Additionally, MR plots of "natural competitors" (i.e. distribution families with two shape parameters, similar range of skewness and kurtosis and/or existing moment-generating function) like the skew Laplace normal (SNL) distribution of Reed and Jørgensen (2003), the exponential generalized beta of the second kind (EGB2) distribution discussed by McDonald (1984) or the skew generalized error (SGED) distribution of Theodossiou (1998) were generated. Figure 2 foreshadows the flexibility of the SGSH distribution which is empirically demonstrated in the terminating section.

\section{Application to Exchange Rate Data}

To analyze the goodness-of-fit of the SGSH distribution, we focus on the daily noon spot US dollar exchange rates (USD/local currency) for the Japanese YEN (YEN) over the period 1 January 1990 to 31 December 2004 (3774 observations) which are available 

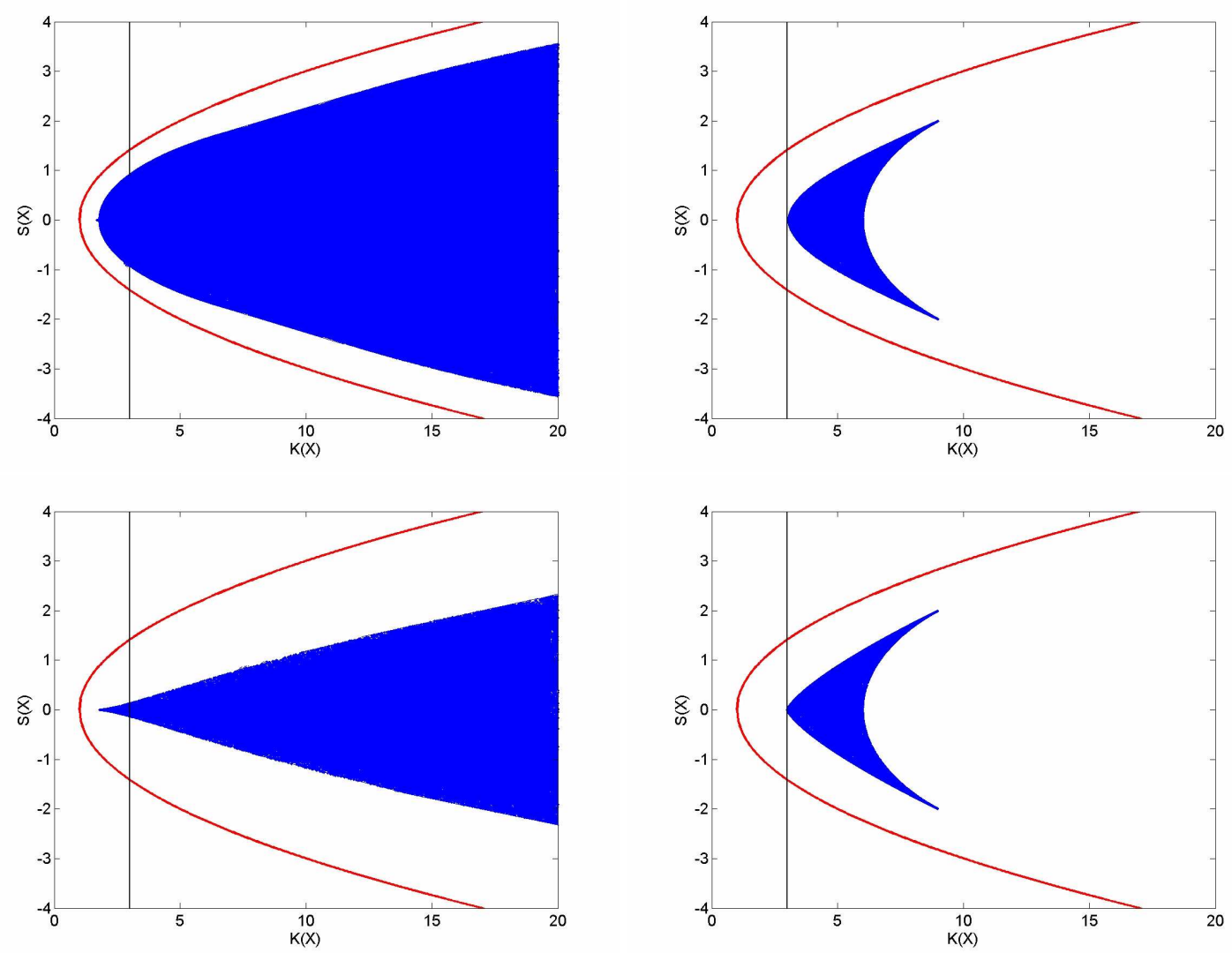

Figure 2: Moment-ratio plots. (a) above/left SGSH, (b) above/right EGB2, (c) below/left SGED, (d) below/right SNL distribution.

from the PACIFIC Exchange Rate Service ${ }^{1}$. In a first step, the exchange rates $S_{t}$ are transformed to percentual log-returns defined as $R_{i, t} \equiv 100 \log \left(S_{t} / S_{t-1}\right)$. Both prices and log-returns can be seen in figure 3 , below.
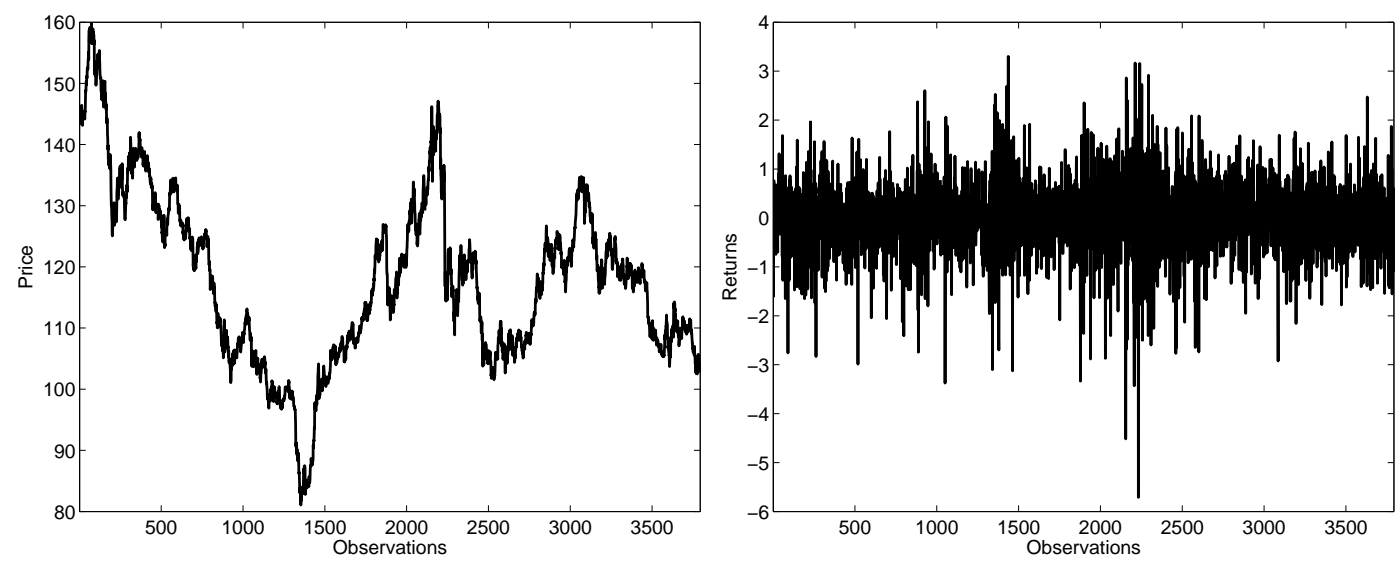

Figure 3: Exchange Rates Dollar/YEN. Prices left and Returns on the right.

\footnotetext{
${ }^{1}$ Download under the URL-link http://pacific. commerce.ubc.ca
} 
The (sample) mean of the log-returns is -0.0093 with a (sample) standard deviation of 0.70 . Moreover, the data set exhibits significant skewness (the skewness coefficientmeasured by the third standardized moments-is given by by -0.5117$)$, whereas the kurtosis coefficient-in terms of the fourth standardized moments-is 7.05 , reflecting the remarkable leptokurtosis.

In order to assess the flexibility of the skew GSH family, we fitted a SGSH distribution (using Maximum-Likelihood estimation) to the exchange rate data and calculated the log-Likelihood value (as an overall measure for the goodness-of-fit). Additionally, the corresponding log-Likelihoods were determined for the skew generalized error distribution (SGED) of Theodossiou (1998), the skew Laplace-normal distribution of Reed and Jørgensen (2003) and exponential generalized beta of the second kind (EGB2) discussed by McDonald (1984). The results are summarized in Table 1, below. Again, the SGSH distribution outperforms the three other parametric models (all of which exhibit lower log-Likelihood values than the SGSH distribution).

Table 1: Maximum-Likelihood estimation: Results.

\begin{tabular}{cccccc}
\hline Distribution & NORM & SGED & EGB2 & SNL & SGSH \\
\hline Log-Likelihood value & -4038.5 & -3817.9 & -3811.7 & -3811.8 & $\mathbf{- 3 8 1 0 . 6}$ \\
\hline
\end{tabular}

\section{Summary}

Within this work we introduced a skew version of Vaughan's (2002) generalized secant hyperbolic distribution by means of exponential tilting. It was shown that all moments exist and that the moment-generating function admits a closed form. Finally, both moment ratio plot and application application to foreign exchange rate data demonstrate the flexibility of our new parametric distribution model.

\section{References}

Elderton, W. P., und Johnson, N. L. (1969). Systems of Frequency Curves. Reading: Addison Wesley.

Esscher, F. (1932). On the probability function in the collective theory of risk. Skandinavisk Aktuarietidskrift, 15, 175-195.

Gerber, H. U., und Shiu, S. W. (1995). Option pricing by esscher transformations. Transactions of the Actuarial Society, 46, 99-191.

McDonald, J. B. (1984). Some generalized functions for the size distribution of income. Econometrica, 52, 647-663.

Reed, W. J., und Jørgensen, M. (2003). The double pareto-lognormal distribution a new parametric model for size distributions. Working Paper. (Department of Mathematics and Statistics, Hamilton, New Zealand) 
Theodossiou, P. (1998). Financial data and the skewed generalized t distribution. Mathematical Science, 44, 1650-1660.

Vaughan, D. C. (2002). The generalized hyperbolic secant distribution and its application. Communications in Statistics - Theory and Methods, 31, 219-238.

Authors' address:

Matthias Fischer

Department of Statistics and Econometrics

University of Erlangen-Nürnberg

90419 Nürnberg, Germany

E-mail: matthias.fischer@wiso.uni-erlangen.de 